\title{
Relation of Matter Wave Verified in Diffusion Theory
}

\author{
Takahisa Okino \\ Department of Applied Mathematics, Faculty of Science \& Engineering, \\ Oita University, 700 Danoharu, Oita City, Oita Prefecture, Japan
}

E-mail addresses: okino@oita-u.ac.jp

Received Sept 2019

Received in revised: Sept 2019

Published: Sept 2019

\begin{abstract}
ABSTRA CT
Investigating the elementary process of diffusion yielded the universal expression of diffusivity relevant to the angular momentum of each microparticle in a material. Using the diffusivity obtained then for the diffusion equation, the wave equation of Schrödinger was theoretically derived from the physical concept in Newton mechanics. The derivation itself reveals that any moving microparticle has the wave image as an intrinsic nature. It was theoretically revealed that the relation having been accepted as a hypothesis proposed by De Broglie is valid for any moving microparticle and that another relation of matter-wave is possible. The new diffusion theory based on the matter-wave will be useful for further development of the nanotechnology in materials science.
\end{abstract}

Keywords: De Broglie hypothesis, Schrödinger equation, Markov process, Diffusion equation

C2019 The Authors. Published by Fundamental Journals. This is an open access article under the CC BY-NC https://creativecommons.org/licenses/by-nc/4.0/

https://doi.org/10.14331/ijfps.2019.330131

\section{INTRODUCTION}

The collective motion of micro particles in a material is governed by the partial differential equation of diffusion in the category of Newton mechanics, and their general solutions (Okino, 2011, 2012, 2015, 2019) depend on the behavior of diffusivity. On the other hand, the motion of single micro particle is governed by the wave equation in the quantum mechanics (Schrödinger, 1926).
The diffusion equation shows that the diffusivity depends on behavior of a micro particle in material. It is thus considered that the quantum effect should be incorporated into the diffusivity. Nevertheless, the diffusivity has been accepted as a proportional factor of the concentration gradient to the diffusion flux called the Fick first law in the existing diffusion theory (Fick, 1855). The discussion about the Markov process theoretically revealed (AA Markov, 1960) that the universal expression $D=\hbar / 2 m$ of diffusivity $D$ is valid for any micro 
particle with a mass $m$ and a momentum $p$ in a local space (Okino, 2013, 2018), using a characteristic constant called the Planck constant $h(=2 \pi \hbar) \mathfrak{m}$ (Planck, 1900). In relation to the derivation of $D=\hbar / 2 m$, it is theoretically revealed that the relation $\lambda=h / p$ having been accepted as a hypothesis proposed by De Broglie is really valid for the wave length $\lambda$ of any moving micro particle (De Broglie, 1923). Using the Boltzmann constant $k_{\mathrm{B}}$ and a correction term $\varepsilon$, the relation of $\lambda=h / \sqrt{m\left(k_{\mathrm{B}} T+\varepsilon\right)}$ is also theoretically derived as another matter wave relation from using the equipartition law of energy (Boltzmann, 1872) for a micro particle in material at an absolute temperature $T$. The elementary process of diffusion depends on the relation $\lambda=h / \sqrt{m\left(k_{\mathrm{B}} T+\varepsilon\right)}$. The concept of wave nature is thus reasonably incorporated into the diffusion mechanism. Further, using the diffusivity $D=\hbar / 2 m$ for the diffusion equation, the wave equation of Schrödinger was theoretically derived from investigating the elastic collision problem in the category of Newton mechanics (Okino, 2013 , 2018), while Schrödinger derived the wave equation from the prior condition of the De Broglie hypothesis. The present derivation itself gives evidence that any moving micro particle has a wave nature because of deriving the wave equation of Schrödinger from the concept of Newton mechanics for any micro particle. Thus, the diffusion theory bridges the gap of physical concept between the Newton mechanics and the quantum mechanics.

The diffusion theory would originally play an important role in the construction of quantum theory if we noticed the diffusivity $D=\hbar / 2 m$ in the period from the energy hypothesis of Planck (Planck, 1900) to the derivation of Schrödinger equation (Schrödinger, 1926). The importance of diffusion theory should be thus reevaluated in the fundamental physics. At the same time, the new diffusion theory discussed here will be useful for a basis of the nanotechnology in materials science. It is one of the aims in the present work that the importance of diffusion theory in physics and also the new fundamental findings obtained here are universally known.

\section{HISTORY IN CLASSICAL QUAMTUM THEORY}

In 1900, the relation $E_{n}=n h v(n=1,2, \cdots)$ of energy hypothesis was proposed by Planck using the frequency $v$ of light in relation to the black body radiation. The Planck relation producing the concept of discrete energy was applied to the phenomena of photo-electric effect (Einstein, 1905). The concept of energy quantum has been thus widely accepted since then. Bohr proposed the relation given by $L_{n}=r_{n} p=n \hbar$, $(n=1,2, \cdots)$ of angular momentum as a model of hydrogen atom, where the electron moves with a momentum $p$ on the circular orbit of discrete radius $r_{n}=r_{n-1}+\Delta r$ (Bohr, 1913). De Broglie proposed that the relation of

$$
p=h / \lambda
$$

is valid between a velocity $v$, a wave length $\lambda$ and a mass $m$ of a micro particle in relation to the momentum $p=m v$ (De Broglie, 1923). Further, the so-called Schrödinger equation was derived in 1926 from accepting the relation of matter wave called the De Broglie hypothesis. Based on the fundamental theory in physics, the relation $|L\rangle=|r \times p\rangle=\mid$ const. $\rangle$ of conservation law of angular momentum $|L\rangle$ is generally valid between a position vector $|r\rangle$ and a momentum $|p\rangle(=m|v\rangle)$ for any micro particle of mass $m$ moving with a velocity $v$ around a central point in a central force field. Here, the notation $\langle r|=| r\rangle^{\dagger}=(x, y, z)$ is the well-known Dirac bracket, and $\langle\tilde{L}|,\langle\tilde{v}|$ and $\langle\tilde{\nabla}|$ used later are $\langle\tilde{L}|=-| L\rangle^{\dagger},\langle\tilde{v}|=-| v\rangle^{\dagger}$ and $\langle\tilde{\nabla}|=-| \nabla\rangle^{\dagger}$ in terms of the Hermite conjugate relevant to the differential operators. For the angular momentum between the orbital radius $r=r_{n}$ and $r=r_{n-1}$ in a central force field, the general relation of

$$
\Delta L=\sqrt{\langle\Delta \tilde{L} \mid \Delta L\rangle}=\| \Delta r \times p\rangle \mid=\Delta r p=l
$$

is valid. Here, $l$ is a constant value relevant to the angular momentum and the relations of

$$
\left\{\begin{array}{l}
|r \times \Delta p\rangle=|\Delta r \times \Delta p\rangle=0, \\
|\Delta L\rangle=\left|r_{n} \times p\right\rangle-\mid\left(r_{n}-\Delta r\right) \times(p-\Delta p\rangle
\end{array}\right.
$$

are used. In the Bohr model, the relation $\Delta L=\hbar \quad l=\hbar$ corresponds to the empirical equation of Balmer in relation to the radiation energy (Balmer, 1885). Further, the experimental results obtained by (Davisson \& Germer, 1928; Frank \& Hertz, 1914; Thomson \& Reid, 1927) show that the relation $2 \pi \Delta r=\lambda$ is valid in a local space between the neighboring discrete orbits in relation to the wave nature of electron.

In those days, we could not understand the reason why the electron does not emit the radiation energy during an acceleration motion in the Bohr model. In the derivation process of the Schrödinger equation discussed later, it is revealed that the concept of acceleration relevant to any micro particle in a local space disappears essentially from the Newton mechanics resulting from the impossibility of identification between two micro particles of the same kind in a local space. We understand the reason mentioned here for the present as follows. It is reasonably accepted that the electron having no concept of acceleration does not emit the radiation energy, even if the electron with an electronic charge moves on the circular orbit in the local space of hydrogen atom. There is no other negative finding relevant to the relation of angular momentum and the relation of wave nature for the orbital electron in the Bohr model. Thus, there is now no doubt that the relations of

$$
\Delta r p=\hbar \text { and } 2 \pi \Delta r=\lambda
$$

are valid for the orbital electron in the Bohr model. Here, equation (3) is just the necessary and sufficient condition for the validity of $p=\hbar / \lambda$. In other words, eliminating $\Delta r$ of (3) reveals that equation (1) is theoretically valid for the orbital electron in Bohr model. Here, we can reconsider the relations of $\Delta r p=\hbar$ and $2 \pi \Delta r=\lambda$ in a local space in the different viewpoint from the orbital electron in the Bohr model as follows. The relation $2 \pi \Delta r=\lambda$ relevant to the orbital radius is also accepted as $k=2 \pi / \lambda=1 / \Delta r$ in a local space in relation to 
the wave number vector $|k\rangle$ in the phase of progressive wave function. The relation $\Delta r p=\hbar$ relevant to the angular momentum is also accepted as $k=p / \hbar=1 / \Delta r$ in relation to the wave number vector. Equation (1) is also valid resulting from eliminating $\Delta r$ from the relations $k=2 \pi / \lambda=1 / \Delta r$ and $k=p / \hbar=1 / \Delta r$, even if the electron moves in a local space considered as a non-central force field. Based on matters mentioned above, it was reasonably revealed that the moving electron has the universal relation $p=h / \lambda$ as an intrinsic nature of the electron itself.

However, it is still uncertain whether or not the relation $p=h / \lambda$ is also theoretically valid for such a micro particle as atom and molecule, even if it is experimentally revealed that such a micro particle has a characteristic nature like a wave image (Arndt, Nairz, Voss-Andreae, \& Keller, 1999; Doak et al., 1999), and (Shimizu, 2001). That is just the reason why the relation $p=h / \lambda$ is still called the hypothesis of De Broglie. In other words, we have now no physical evidence that the theoretical equation $p=h / \lambda$ is valid for any moving micro particle. It seems that the diffusion theory has no connection with matters mentioned above. In fact, we cannot find such a discussion. In the following, however, investigating the elementary process of diffusion will reveal that the diffusion theory plays an important role for understanding the physical essence of matters mentioned above.

\section{ELEMENTARY PROCESS OF DIFFUSION}

In the following, the importance of diffusion theory in the fundamental physics will be revealed. In that case, for such a micro particle as atom and molecule having an internal structure, the internal structure is neglected in the present work.

\section{Angular momentum of micro particle in a local space}

The proof of relation $\Delta r p=\hbar$ valid for any micro particle was too simplified in the previous works (Okino, 2013, 2018). In the following, therefore, it is described in detail that the relation $\Delta r p=\hbar$ is theoretically valid for any micro particle in a local space, even if any micro particle moves in the field of non-central force.

Based on the fact that the moving electron is governed by (1) as revealed in the previous Chapter, the angular momentum $\Delta L=\Delta r p$ for any micro particle is discussed in the following. The electron incident on the surface of a material is not captured by any vacant local space because of nonexistence of a large central force like the case of Bohr model. The electron thus moves through interstices between micro particles in the material at an absolute temperature $T$, repeating collisions with the micro particles. In that situation, the equipartition law of energy relevant to a group of micro particles indicates that the velocity $v^{*}$ of electron with a mass $m^{*}$ is given by $v^{*}=\sqrt{\left(k_{\mathrm{B}} T+\varepsilon\right) / m}$ per a degree of freedom, where $\varepsilon$ is a correction term due to the uncertainty principle for the equipartition law of energy at $T=0$. The relation obtained here results in the correlation given by

$$
\left\{\begin{array}{l}
v^{*}=\sqrt{\left(k_{\mathrm{B}} T+\varepsilon\right) / m^{*}} \\
\Delta r^{*}=\sqrt{\left(k_{\mathrm{B}} T+\varepsilon\right) / m^{*}} \Delta t^{*}
\end{array} \rightarrow \Delta r^{*} p^{*}=\hbar=\left(k_{\mathrm{B}} T+\varepsilon\right) \Delta t^{*},\right.
$$

where a value of time $\Delta t^{*}$ is a characteristic one dependent only on $T$.

Here, note that the relation $\hbar=\left(k_{\mathrm{B}} T+\varepsilon\right) \Delta t^{*}$ has no intrinsic quantity relevant to the electron, although it is derived from the matter relevant to the electron. The relation $\hbar=\left(k_{\mathrm{B}} T+\varepsilon\right) \Delta t^{*}$ is considered to be universal for any micro particle in a material. In that case, it is considered that the characteristic value of $\Delta t^{*}$ is relevant to the frequency $v$ of the harmonic oscillator in relation to the equipartition law of energy. Thus, the correlation of

$$
\left\{\begin{array}{l}
\Delta t^{*}=\hbar /\left(k_{\mathrm{B}} T+\varepsilon\right) \\
1 / v=2 \pi \Delta t^{*}
\end{array} \rightarrow h v=k_{\mathrm{B}} T+\varepsilon\right.
$$

may be valid in the present case. For any micro particle with a mass $m$ in the same material at the temperature $T$ used above for the electron, the relation of $v=\sqrt{\left(k_{\mathrm{B}} T+\varepsilon\right) / m}$ is also valid in the same manner. The relation $\Delta r p=\left(k_{\mathrm{B}} T+\varepsilon\right) \Delta t$ should be also theoretically valid then. The relation $\Delta t^{*}=\Delta t$ is valid then because of the dependence only on $T$. Judging from the relation $\Delta t^{*}=\Delta t$, the relations of

$$
\left\{\begin{array}{l}
\Delta r^{*} p^{*}=\left(k_{\mathrm{B}} T+\varepsilon\right) \Delta t^{*}=\hbar, \\
\Delta r p=\left(k_{\mathrm{B}} T+\varepsilon\right) \Delta t
\end{array}\right.
$$

result in the universal relation of

$$
\Delta r p=\hbar,
$$

which is theoretically valid for any moving micro particle in a material.

\section{Universal expression of diffusivity}

For the concentration $C(t, x, y, z)$ dependent on the time and space coordinate setting the origin at a center point of local space in a material, the Markov process shows that the relation given by

$$
C(t+\Delta t, x, y, z)=\frac{1}{2}\left\{\begin{array}{l}
C(t, x+\Delta x, y+\Delta y, z+\Delta z) \\
+C(t, x-\Delta x, y-\Delta y, z-\Delta z)
\end{array}\right\}
$$

is valid in the elementary process of diffusion. The Taylor expansion of (5) yields

$$
\partial_{t} C=D\langle\tilde{\nabla} \mid \nabla\rangle C \quad \text { for } \quad(\Delta r)^{2} / 2 \Delta t \rightarrow D,
$$

where the abbreviated notation $\partial_{t}=\partial / \partial t$ is used. Here, it is physically accepted that the value of $(\Delta r)^{2} / 2 \Delta t$ in each local space in a material converges to a statistical mean value $D=(\Delta r)^{2} / 2 \Delta t$ called the diffusivity. It is reasonable that a micro particle exists at the center $r=0$ in the time average at the absolute temperature $T=0$. If a micro external force acts 
on the micro particle, it moves from $r=0$ to $r=\delta$ for $0<\delta<<1$ then. Subsequently, it returns to $r=0$ again, if the force is removed. This means that the averaged centripetal force caused by micro particles around the concerned micro particle acts on the micro particle itself in the local space. Substituting equation (4) into the diffusivity expression $D=(\Delta r)^{2} / 2 \Delta t=\Delta r p / 2 m$ using a momentum $p$ results in the universal diffusivity of

$$
D=\Delta r p / 2 m=\hbar / 2 m
$$

in the averaged central force field mentioned above, where the temperature dependence is neglected.

\section{Derivation of Schrödinger equation}

Since Schrödinger used the well-known Euler relation $e^{i x}=\cos x+i \sin x$ instead of the trigonometric function for the calculation in the derivation process, the so-called Schrödinger equation becomes the partial differential equation for a complex-value function. The Schrödinger equation for a complex-value function $\psi$ in the free field is expressed as

$$
i \hbar \partial_{t} \psi=\frac{\langle\tilde{p} \mid p\rangle}{2 m} \psi .
$$

An elastic collision problem between micro particles A and B of the same kind was investigated in the category of Newton mechanics (Okino, 2013). The particle $A$ moving with a velocity $v_{\mathrm{A}}=v_{0}$ collides at the time $t=0$ with the particle $B$ in the standstill state in the initial state (a). If we can make the discrimination between $A$ and $B$ just after the collision between $0 \leq t \leq \Delta t<<1$, the particle A decelerates from the velocity $v_{\mathrm{A}}=v_{0}$ to the velocity zero and the particle $\mathrm{B}$ accelerates from the velocity zero to the velocity $v_{\mathrm{B}}=v_{0}$ in the collision process (b). On the other hand, if we cannot make the discrimination between $A$ and $B$ just after the collision, it seems that the particle A decelerates from the velocity $v_{\mathrm{A}}=v_{0}$ to the velocity zero between $0<t \leq \varepsilon$ and subsequently accelerates again from the velocity zero to the velocity $v_{\mathrm{A}}=v_{0}$ between $\varepsilon \leq t \leq \Delta t$ in the present case (c). In other words, the motion of particle A then seems as if there is no collision process.

We generally discriminate between $A$ and $B$ by means of observing the reflected visible light of wave length $\lambda$ caused by the collision between the incident light and micro particles $A$ and $B$. For the distance $d$ between $A$ and $B$, it is necessary for the discrimination to hold the condition of $\lambda<d$. Here, the relation $E=h c / \lambda$ of photon energy indicates that a large energy of $E$ is necessary for a small value of $d$ because of $\lambda<d$ then. When a value of $d$ is small, we cannot theoretically discriminate between $\mathrm{A}$ and $\mathrm{B}$ because of the disturbance caused by the large $E$ due to small $\lambda$. In the space $|r\rangle=(x, y, z)^{\dagger}$, the acceleration $|a\rangle$ is defined as;

$$
|a\rangle=\partial_{t}^{2}|r\rangle=\left\{\lim _{\Delta t \rightarrow 0} \frac{\Delta}{\Delta t}\right\}^{2}|r\rangle
$$

In the above latter case (c), the change of acceleration from $a=-a_{\mathrm{A}}$ between $0<t \leq \varepsilon$ to $a=a_{\mathrm{A}}$ between $\varepsilon \leq t \leq \Delta t$ indicates that the impossibility of identification between the particles $A$ and $B$ just after the collision yields the transformation of differential operator from $\partial_{t}=\lim _{\Delta \rightarrow 0} \Delta / \Delta t$ between $0<t \leq \varepsilon$ to $\pm i \lim _{\Delta t \rightarrow 0} \Delta / \Delta t= \pm i \partial$, between $\varepsilon \leq t \leq \Delta t$, as can be seen from (9). The impossibility of identification mentioned here thus indicates the necessity of imaginary differential operators for the diffusion equation. Therefore, we mathematically accept the imaginary operator $\pm i \partial_{t}$ as an essential characteristic caused by the impossibility of identification. The concept of acceleration in Newton mechanics then disappears from the present collision problem, although that of $\Delta t|a\rangle$ or a velocity $|v\rangle$ is still meaningful for the limit of $\Delta t \rightarrow 0$. Further, $|\nabla\rangle \rightarrow \pm i|\nabla\rangle$ is also valid in accordance with $\partial_{t} \rightarrow \pm i \partial_{t}$ because of

$$
\partial_{x}=\lim _{\Delta x \rightarrow 0}\left\{\frac{\Delta}{\Delta x}\right\}=\lim _{\Delta t \rightarrow 0}\left\{\frac{\Delta t}{\Delta x} \frac{\Delta}{\Delta t}\right\}=\left(1 / v_{x}\right) \partial_{t} .
$$

The progressive wave function is generally expressed as $f=A \exp [i(\langle k \mid r\rangle-\omega t)]$ by using a wave number $k=2 \pi / \lambda$ and an angular velocity $\omega=2 \pi v$. In relation to a circular motion of radius $r_{n}=r_{n-1}+\Delta r, \quad \lambda=2 \pi \Delta r \quad$ or $\quad k=1 / \Delta r$ corresponds to $v=1 / 2 \pi \Delta t$ or $\omega=1 / \Delta t$ in the progressive wave function. If we take account of the eigenvalues of the imaginary differential operators for the progressive wave function, the signs of $\partial_{t} \rightarrow \pm i \partial_{t}$ and $|\nabla\rangle \rightarrow \pm i|\nabla\rangle$ should be physically determined as

$$
\left\{\begin{aligned}
& \partial_{t} \rightarrow i \partial_{t}=\lim _{\Delta t \rightarrow 0}\{\Delta /(-i \Delta t)\}, \\
&|\nabla\rangle \rightarrow-i|\nabla\rangle=\left\langle\lim _{\Delta x \rightarrow 0}\left\{\frac{\Delta}{i \Delta x}\right\}, \lim _{\Delta y \rightarrow 0}\left\{\frac{\Delta}{i \Delta y}\right\},\left.\lim _{\Delta z \rightarrow 0}\left\{\frac{\Delta}{i \Delta y}\right\}\right|^{+} .\right.
\end{aligned}\right.
$$

Using the diffusivity of (7) and the imaginary differential operators discussed here for the elementary process of diffusion, equation (6) is rewritten as

$$
i \partial_{t} C=-\{\hbar / 2 m\}\langle\tilde{\nabla} \mid \nabla\rangle C
$$

Here, note that the concentration $C$ obtained by solving (10) becomes a complex-value function then, as can be seem from the solution obtained by using the usual method of separation of variables for (10). Using the imaginary differential operators mentioned above for $\lambda=2 \pi \Delta r$ of momentum $p=h / \lambda=\hbar k$ and $v=1 / 2 \pi \Delta t$ of photon energy $E=h v=\hbar \omega$, the well-known basic operators in quantum mechanics are obtained as

$$
\left\{\begin{array}{l}
p_{x}=\frac{h}{\lambda_{x}}=\frac{\hbar}{\Delta x} \rightarrow \hbar \lim _{\Delta x \rightarrow 0} \frac{\Delta}{i \Delta x}=-i \hbar \partial_{x}, \\
E=h v=\hbar \frac{1}{\Delta t} \rightarrow \hbar \lim _{\Delta t \rightarrow 0} \frac{\Delta}{-i \Delta t}=i \hbar \partial_{t} .
\end{array}\right.
$$

Using equation (11) for (8), the Schrödinger equation is thus rewritten as

$$
i \hbar \partial_{t} \psi=-\left\{\hbar^{2} / 2 m\right\}\langle\tilde{\nabla} \mid \nabla\rangle \psi
$$


Here, the Schrödinger equation of (12) is also reasonably obtained in the category of Newton mechanics by rewriting the complex-value function like $C \rightarrow h \psi$ in equation (10). Regardless of the De Broglie hypothesis used by Schrödinger for the derivation of (12), the same equation was theoretically derived from investigating the elementary process of diffusion. The derivation itself discussed here is meaningful in the fundamental physics, since the analytical method used here bridges the gap of physical concept between the Newton mechanics and the quantum mechanics. In particular, the fact that the wave equation of Schrödinger was theoretically derived from the diffusion equation relevant to the Brown motion in the category of Newton mechanics gives the remarkable and important evidence that any moving micro particle in a local space has the wave image given by

$$
\lambda=2 \pi \Delta r
$$

It was thus for the first time theoretically revealed that any moving micro particle has the wave nature. In addition, the wave nature of some micro particles like atom or molecule is also confirmed by the experimentation (Arndt et al., 1999; Doak et al., 1999) and (Shimizu, 2001)

\section{Relation of matter wave}

As discussed above, equations (4) and (13) corresponding to the necessary and sufficient condition of (3) have been already obtained. We can thus conclude that equation (1) is universally valid as an intrinsic nature of any moving micro particle in a material. Hereafter, equation (1) should be recognized as not a hypothesis but a real one valid theoretically in physics. Here, substituting equation (1) into the relation $p=m v=\sqrt{m\left(k_{\mathrm{B}} T+\varepsilon\right)}$ relevant to the equipartition law of energy yields

$$
\lambda=h / \sqrt{m\left(k_{\mathrm{B}} T+\varepsilon\right)}
$$

Equation (14) is considered as another relation of matter wave in a material at an absolute temperature $T$. The limit of $m \rightarrow \infty$ in the relation of (14) indicates that the quantum characteristic of matter wave disappears from the particle concerned. Equation (14) is applicable to investigate behavior of any moving micro particle in a material. The diffusion mechanism just corresponds to (14) acceptable as another relation of matter wave.

\section{New diffusion mechanism}

It is well-known that a micro particle in a crystal material is not in the standstill state but in the lattice vibration or thermal vibration state (Born \& Huang, 1954). On the other hand, equation (7) indicates that a micro particle in material moves on a fixed circular orbit on the spherical surface of local space because of the conservation law of angular momentum. The great circular motion of micro particle on the orbital plane perpendicular to the $z$ axis corresponds to the simple harmonic motion in the direction of $x$ or $y$ axis. Thus, the lattice vibration or thermal vibration of micro particle in a material corresponds to the simple harmonic motion mentioned here. A lattice constant for a crystal material or a mean distance between neighboring micro particles for an amorphous material is denoted as $a$ at a given temperature $T$.
If equation (14) satisfies the relation $\lambda \leq 2 \pi a$, the micro particle with mass $m$ is able to exist in the local space. It is considered then that such micro particle is stable under the condition of radius $r=r_{n}=n \lambda / 2 \pi \quad(n=1,2, \cdots)$ for $r_{n} \leq a$ in a central force field. We conceive in the following that a micro particle with mass $m=m_{\mathrm{P}}$ forming the population or a micro particle with mass $m=m_{j}$ in a material makes a great circuit of radius $r=r_{n}$ on the $z=0$ plane in a local space.

(i) In case of $h^{2} / a^{2}\left(k_{\mathrm{B}} T+\varepsilon\right) \leq m_{j} \leq m_{p}$

The micro particle is able to occupy the local space between $\lambda_{\mathrm{p}} / 2 \pi \leq r \leq a$ in the present case. The motion of micro particle is stable as a wave image under the condition of $r=r_{n}=n \Delta r$, for $(n=1,2, \cdots)$ but $n \leq 2 \pi a / \lambda_{\mathrm{p}}$ and $\Delta r=\lambda_{j} / 2 \pi$. In the state of $r=r_{n}+\delta$ for $\delta<\Delta r$ caused by a thermal fluctuation, however, the micro particle (quantum) of a wave image becomes unstable and it then jumps in an instant from the local space to a near vacant local space. Such a quantum moves through a path composed of interstices between micro particles in a material until the quantum collides with a micro particle on the path. In that situation, the movement of quantum is thus translator one with the wave number $k=p / \hbar=1 / \Delta r$ and equation (1) is still valid then because of the relation $\lambda_{j}=h / \sqrt{m_{j}\left(k_{B} T+\varepsilon\right)}$ of wave nature.

(ii) In case of $m_{j}>m_{\mathrm{p}}$

The relation $\lambda_{j}=h / \sqrt{m_{j}\left(k_{B} T+\varepsilon\right)}$ in the present case indicates that the micro particle with mass $m_{j}$ makes a circuit of radius between $0<r<\lambda_{\mathrm{p}} / 2 \pi$ as a wave image in the central force field. Such a micro particle may be considered as an impurity particle in the concerned material. Such impurity particle is relatively large in size compared with the micro particle of population. Nevertheless, the orbital radius $r=r_{n}$ becomes relatively easily in the state of $r=r_{n}+\delta$ in terms of a thermal fluctuation because of a large momentum $p_{j}=\sqrt{m_{j}\left(k_{B} T+\varepsilon\right)}$. It is thus able to jump from the local space to a near vacant local space through the path composed of the interstices in material as a wave image in spite of the micro particle of relatively large size.

(iii) In case of $m_{j}<\hbar^{2} / a^{2}\left(k_{\mathrm{B}} T+\varepsilon\right)$

In the present case, a micro particle relatively small in size compared with the micro particle of population is unable to occupy a local space in material because of $r>a$. Such micro particle may be also considered as an impurity particle. As a result, it diffuses at random through the interstices in material as a wave image, repeating collisions with micro particles. The theory of elementary process of diffusion mentioned above shows that the progress of diffusion does not always depend on the existence of neighboring vacant local space. However, the existence of neighboring vacant local space affects on the diffusion progress. In general, the conservation law of angular momentum is valid for each micro particle in the above case (i) or (ii). The orbital plane is immovable as far as $(d / d t)\left|L_{n}\right\rangle=0$ is valid. Each unit vector perpendicular to an orbital plane of micro particle is different from each other then. In other words, jumping possibility and also jumping direction 
of a micro particle depend on a spatial correlation between the unit vector and the neighboring vacant local space. It is an extremely low probability that a path of composed of the interstices continues from the surface of thin film to the back. When that happened, such a micro particle incident on the surface of thin film as one satisfying $a \sqrt{m_{j}\left(k_{B} T+\varepsilon\right)}<\hbar$ moves to the back directly through the path as a quantum with a wave image then. That is considered as the tunnel effect in the quantum mechanics.

\section{DISCUSSION AND CONCLUSION}

From a viewpoint of the fundamental physics, it was theoretically revealed that the diffusion theory plays an important role for the establishment of quantum theory. At the same time, it was found that the equation $\lambda=\hbar / p$ of the matter wave having been accepted as a hypothesis for a long time and further another equation $\lambda=h / \sqrt{m\left(k_{\mathrm{B}} T+\varepsilon\right)}$ derived from the present work are theoretically valid for any moving micro particle as a fundamental equation in physics. As a result, the development from the Newton mechanics into the quantum mechanics was produced from what the discrimination between two micro particles of the same kind moving in a local space is principally impossible.

For the Brown motion (Brown, 1828), Einstein revealed evidence that a material is composed of atoms or molecules by deriving the diffusivity expression (Einstein, 1905).The Fick first law $|J\rangle=|v\rangle C$ expressed by a velocity $|v\rangle$ and a concentration $C$ of Brown particles was used for the calculation then. Substituting the relation $|J\rangle=|v\rangle C$ into the

\section{REFERENCES}

AA Markov, N. N. (1960). The theory of algorithms. English translation Mathematics and its applications (Soviet series). Kluwer Academic Publishers, Dordrecht, Boston, and London, 1988. The Journal of Symbolic Logic, 56(1), 336337.

Arndt, M., Nairz, O., Voss-Andreae, J., \& Keller, C. (1999). G. van der Zouw, G.; Zeilinger, A. Wave-Particle Duality of C60 Molecules. Nature, 401, 680-682.

Balmer, J. J. (1885). Notiz über die Spectrallinien des Wasserstoffs. Annalen der physik, 261(5), 80-87.

Bohr, N. (1913). XXXVII. On the constitution of atoms and molecules. The London, Edinburgh, and Dublin Philosophical Magazine and Journal of Science, 26(153), 476-502.

Boltzmann, L. (1872). Weitere Studien über das Wärmegleichgewicht unter Gasmolekülen, Sitzungs. Akad. Wiss. Wein 66 (1872), 275-370; English: Further Studies on the Thermal Equilibrium of Gas Molecules. Kinetic Theory, 2, 88-174.

Born, M., \& Huang, K. (1954). Dynamical theory of crystal lattices: Clarendon press.

Brown, R. (1828). XXVII. A brief account of microscopical observations made in the months of June, July and August 1827 , on the particles contained in the pollen of plants; and on the general existence of active molecules in organic and diffusion flux $|J\rangle$ of the continuous equation $\partial_{t} C+\langle\tilde{\nabla} \mid J\rangle=0$ yields not the diffusion equation but the Euler equation $\partial_{t} C+\langle\tilde{v} \mid \nabla\rangle C=0$ in the fluid mechanics (Euler, 1761). It is self-contradictory that the diffusion flux relevant to diffusion phenomena yields the Euler equation relevant to the fluid phenomena. The paradox pointed out here was reasonably solved in relation to the coordinate transformation of diffusion equation (Okino, 2019). It was thus revealed then that equation (6) is a moving coordinate system and corresponds to the usual expression $\partial_{t} C=\langle\tilde{\nabla} \mid D \nabla\rangle C$ of a fixed coordinate system. It was also theoretically revealed then that the Fick laws are not consistent with the condition of law.

There is often such a physically dominant matter that a relation is universally valid under a given condition in accordance with an empirical rule in physics. If we cannot theoretically demonstrate its validity then, it has been accepted as a hypothesis or a law in physics.

We conclude here that the De Broglie hypothesis and the Fick first or second law are not a hypothesis and low but the real equations valid reasonably in physics. On the other hand, the descriptions of these hypothesis and laws are still shown in the existing textbooks concerned. Judging from the educational viewpoint for younger people, the truth of physical theory should be suitably described in the existing textbooks as soon as possible. The importance of diffusion theory should be thus reevaluated in the fundamental physics.

In addition, the new diffusion mechanism in the present work and the basis of diffusion theory revealed in the previous works will be highly useful for a further development of the nanotechnology in materials science.

inorganic bodies. The Philosophical Magazine, 4(21), 161173.

Davisson, C., \& Germer, L. (1928). Reflection of electrons by a crystal of nickel. Proceedings of the National Academy of Sciences of the United States of America, 14(4), 317.

De Broglie, L. (1923). Waves and quanta. Nature, 112(2815), 540.

Doak, R., Grisenti, R., Rehbein, S., Schmahl, G., Toennies, J., \& Wöll, C. (1999). Towards realization of an atomic de Broglie microscope: helium atom focusing using Fresnel zone plates. Physical review letters, 83(21), 4229.

Einstein, A. (1905). Über einen die Erzeugung und Verwandlung des Lichtes betreffenden heuristischen Gesichtspunkt. Annalen der physik, 322(6), 132-148.

Euler, L. (1761). Principia motus fluidorum. Novi commentarii academiae scientiarum Petropolitanae, 271-311.

Fick, A. (1855). V. On liquid diffusion. The London, Edinburgh, and Dublin Philosophical Magazine and Journal of Science, 10(63), 30-39.

Frank, J., \& Hertz, G. (1914). Über die erregung der Quecksilberresonanzlinie 253.6 durch Elektronenstösse. Verhandlungen der Deutschen Physikalischen Gesellschaft, Berlin, 16, 512.

Okino, T. (2011). New Mathematical Solution for Analyzing Interdiffusion Problems. Materials Transactions, 52(12), 2220-2227.

Okino, T. (2012). Brownian Motion in Parabolic Space. Journal of Modern Physics, 3(03), 255. 
Okino, T. (2013). Correlation between Diffusion Equation and Schrödinger Equation. Journal of Modern Physics, 4(05), 612.

Okino, T. (2015). Mathematical Physics in Diffusion Problems. Journal of Modern Physics, 6(14), 2109.

Okino, T. (2018). Quantum Effect on Elementary Process of Diffusion and Collective Motion of Brown Particles. Journal of Modern Physics, 9(05), 1007.

Okino, T. (2019). Establishment of New Fundamental Theory in Diffusion Phenomena. Applied Physics Research, 11(1). doi: 10.5539/apr.v11n1
Planck, M. K. E. L. (1900). Zur theorie des gesetzes der energieverteilung im normalspectrum. Verhandl. Dtsc. Phys. Ges., 2, 237.

Schrödinger, E. (1926). Quantisierung als eigenwertproblem. Annalen der physik, 385(13), 437-490.

Shimizu, F. (2001). Specular reflection of very slow metastable neon atoms from a solid surface. Physical review letters, 86(6), 987.

Thomson, G. P., \& Reid, A. (1927). Diffraction of cathode rays by a thin film. Nature, 119(3007), 890. 